\title{
Reliability analysis of repairable systems using system dynamics modeling and simulation
}

\author{
M. Srinivasa Rao $\cdot$ V. N. A. Naikan
}

Received: 22 May 2014 / Accepted: 31 May 2014/Published online: 24 July 2014

(C) The Author(s) 2014. This article is published with open access at Springerlink.com

\begin{abstract}
Repairable standby system's study and analysis is an important topic in reliability. Analytical techniques become very complicated and unrealistic especially for modern complex systems. There have been attempts in the literature to evolve more realistic techniques using simulation approach for reliability analysis of systems. This paper proposes a hybrid approach called as Markov system dynamics (MSD) approach which combines the Markov approach with system dynamics simulation approach for reliability analysis and to study the dynamic behavior of systems. This approach will have the advantages of both Markov as well as system dynamics methodologies. The proposed framework is illustrated for a standby system with repair. The results of the simulation when compared with that obtained by traditional Markov analysis clearly validate the MSD approach as an alternative approach for reliability analysis.
\end{abstract}

Keywords Simulation approach - Reliability analysis · Dynamic implications of standby systems · Markov system dynamics approach $\cdot$ Complex systems

\footnotetext{
M. Srinivasa Rao $(\bowtie)$

Department of Mechanical Engineering, GMR Institute of

Technology, Rajam, India

e-mail: srinivas.m@gmrit.org

V. N. A. Naikan

Reliability Engineering Centre, Indian Institute of Technology,

Kharagpur, India

e-mail: naikan@hijli.iitkgp.ernet.in
}

\section{Introduction}

In the dynamic and highly competitive business environment, manufacturing industries are under tremendous pressure due to the free market economy, rapid technological development, and continuous changes in customer demands. These demands emphasize the need for high levels of overall system reliability that include the reliability of all human elements, machines, equipment, material handling systems and other value-added processes and management functions throughout the manufacturing system. An organization needs to identify the characteristics of the various disturbances and their consequential effects over time. It is basically a giant network of interconnected nodes. Changes in one part of an organization can affect other parts of the organization with surprising and often negative consequences (Islam and Tedford 2012).

In today's technological world, almost every person depends upon the continued carrying out of a broad array of compound machinery, equipments and services for our everyday safety, security, mobility and economic welfare. The concept of failure rate is vital in reliability and survival analysis. Nevertheless, obtaining the failure rate in numerous practical situations is regularly not so simple, as the structure of the system to be considered, for instance, can be rather complex or the process of the failure development cannot be explained in the simple way. In these cases, an appropriate model can help a lot in deriving reliability characteristics. Time domain modeling is concerned with the behavior of system reliability over time. The simplest time-dependent failure model assumes that failures arrive randomly with inter-arrival times exponentially distributed with constant rate. Generally, researchers organize their reliability analysis via using exponential 
cumulative distribution function when failure rate has a constant rate (Raissi and Gatmiry 2012).

The study and analysis of repairable standby systems is an important topic in reliability. Analytical techniques become very complicated and unrealistic especially for modern complex systems. Traditional techniques cannot effectively deal with the uncertainties of the risk response development. Moreover, decision making process often involves the experts' subjective judgments and preferences regarding qualitative/quantitative criteria for mega projects. This problem may result in imprecise and indefinite data being present, which makes the decision making process complex and challenging. To mitigate these difficulties, Mousavi et al. (2012) introduced a new multiple criteria decision making approach with interval computations to conquer these difficulties for mega projects. The complexity of the modern engineering systems besides the need for realistic considerations when modeling their availability/reliability renders analytical methods very difficult to be used.

Repairable standby systems have attracted the attention of several authors in the field of reliability theory. Various researchers including (Goel et al. 1990) have analyzed twounit cold standby system models assuming that the repair of the failed unit continues until the unit becomes as good as new. Gupta and Goel (1991) analyzed a two-unit (one priority and other ordinary) cold standby system model assuming that each unit has no independent and nonidentical components. It is also assumed that, if the repair of the ordinary unit is not complete within a specified time, known as the maximum repair time, then the ordinary unit is discarded. They have assumed that failure and repair times are uncorrelated. Gupta and Mumtaz (1996) analyzed a two-identical unit cold standby system model, assuming that an order is placed to replace the failed unit by a new one if the unit is not repaired during the specified time limit. Many papers in the field of reliability theory have investigated system models assuming that upon failure of a unit, it is replaced by its standby unit. Moafi et al. (1991) presented a comparison of two models of a system that has two non-identical active series units with identical cold standbys. Mokaddis et al. (1997) have studied the two-unit cold standby redundant system where the repairman is always available to inspect and repair the failed unit, and replace the failed unit with the standby unit if it is available instantaneously. There is extensive literature on availability characteristics of repairable systems with two or three components under varying assumptions on the failures and repairs (Zhang and Lam 1996; Song and Deng 1993; Lal et al. 2005).

In these papers, authors used either Laplace transforms method or Lagrange's method to solve Chapman-Kolmogorov differential equations associated with a particular problem. It has been observed that these methods involve complex computations and it is very difficult to calculate reliability of the system by these methods. And also in most of these papers, exponential distributions are assumed for some system variables and only one repair facility is considered for mathematical convenience. Methods used in the existing literature deals with non Markov systems involving analytical techniques such as regenerative point technique and the supplementary variables techniques which contains many differential equations. However, it is still not easy to solve these differential equations. Therefore, the issues of how to formulate and solve the system of Markov renewal equations and how to specify those undetermined functions are gradually becoming interesting and important in the analysis of stochastic models. Several research papers and books have been published that discuss various facts of reliability technology. This paper is focused on the development of a system dynamics simulation methodology for the reliability analysis of a standby system with repair.

Traditional statistical methods for recognizing and evaluating systems are not always appropriate, especially when enough data are not available. In the real world, the static mathematical models are resulted from a system full of non-deterministic nature; their parameters become nonunique uncertainties and a chain of uncertainties. There are many approaches for accounting for uncertainty and deviation, for example, mathematics models and simulation tools (Khalaj et al. 2013).

Many researchers have been searching for alternate methodologies for more practical and realistic reliability analysis. Simulation has been used as a powerful tool for modeling and analysis of system reliability. It is used to represent the dynamic behavior of systems in the most realistic sense.

A system dynamics representation of Markov models opens up the possibility of numerical solution and of avoiding the tedium of analytical solution. Another advantage of system dynamics modeling is that it is easy to experiment with alternative values of parameters. Hence, sensitivity analysis can be performed easily during reliability estimation and prediction. Finally, the steady state solutions for these problems can be obtained easily only by visual inspection of the flow diagrams and by making use of the fact that the net flow into a level is zero in the steady state.

This paper focuses on a simulation methodology for the reliability analysis of a standby system with repair. The remaining part of the paper is structured as follows. Section 2 gives the modeling aims and approach. Section 3 gives a Markov system dynamics (MSD) approach to system availability/reliability assessment. Section 4 gives the reliability assessment of a standby system with repair. Section 5 gives results and analysis and Sect. 6 gives the concluding comments. 


\section{Modeling aim and approach}

In fact, whereas the problem of determining long run availability of the system has been extensively studied in the literature, the problem of finding the reliability of the system has not satisfactorily been tackled till now. However, among several available methods, Markov method is widely used for reliability analysis. This has motivated us to propose a novel system dynamics simulation technique for reliability analysis. This technique is used to study and analyze the dynamic behavior of systems in the most realistic sense. The present work proposes a hybrid approach called as MSD approach which combines the Markov approach with system dynamics simulation approach to overcome some of the limitations of Markov process in a simple and efficient way for reliability analysis and to study the dynamic behavior of systems as described below. In the present work, initially it has been proved that the stationary, continuous-time Markov models are algebraically equivalent to linear system dynamics models as shown below.

The continuous-time Markov process

- A continuous-time Markov process is completely described by its transition probability function $p_{i j}(t)$ which is the probability that the system is in state $j$ at time $t$ if it was in state $i$ at time 0 .

- And also $p_{i j}(t+\Delta t)=P\{X(t+\Delta t)=j \mid X(0)=$ $i\}=\sum_{k=1}^{S} P\{X(t+\Delta t)=j, X(t)=k \mid X(0)=i\}$

where $S$ is the total number of states that the system can occupy at any time.

Using the Baye's theorem, the above equation can be obtained as follows:

$$
\begin{aligned}
& p_{i j}\{t+\Delta t\}=\sum_{k=1}^{S} P\{X(t+\Delta t)=j \mid X(t)=k, X(0)=i\} \\
& \quad \times P\{X(t)=k \mid X(0)=i
\end{aligned}
$$

Using the Markov's assumption of forgetfulness, the above equation can be obtained as follows:

$$
\begin{aligned}
p_{i j}(t+\Delta t)= & \sum_{k=1}^{S} P\{X(t+\Delta t)=j \mid X(t)=k\} P\{X(t) \\
& =k \mid X(0)=i\}
\end{aligned}
$$

Defining $\lambda_{k j}(t)=P\{X(t+\Delta t=j \mid X(t)=k\}$.

And noting that $P\{X(t)=k \mid X(0)=i\}=p_{i k}(t)$.

So, $p_{i j}(t+\Delta t)=\sum_{k=1}^{S} p_{i k}(t) \times \lambda_{k j}(t)$

This equation is called as the Chapman-Kolmogorov equation. $\lambda_{k j}(t)$ is called the transfer rate from state $k$ to state $j$ at time $t$.

Theoretically, the transfer rates can be time varying or even state-dependent, but in general, they are assumed to be constant. This is the stationarity assumption. Most of the literature on Markov process makes this stationarity assumption and such models are termed as homogeneous (or stationary) Markov models.

- A stationary, continuous-time Markov model is then given by [Eq. (1) becomes as follows],

$p_{i j}(t+\Delta t)=\sum_{k=1}^{S} P_{i k}(t) \times \lambda_{k j}$

Transition probabilities are continuous functions of time with the following properties:

$0 \leq p_{i j} \leq 1 \quad$ for all $i, j$ and $t$

$\sum_{j=1}^{S} p_{i j}(t)=1 \quad$ for $i=1,2, \ldots \ldots \ldots \ldots, S$

and $\lambda_{k j}$ has the following properties:

$\lambda_{k j} \geq 0 \quad$ for $k \neq j$

$\lambda_{k j} \leq 0 \quad$ for $k=j$

$\sum_{j=1}^{S} \lambda_{k j}=0 \quad$ for $k=1,2, \ldots \ldots \ldots, S$

- The rate of change for $p(t)$, where $p(t)$ is a square matrix of transition probabilities, can be written in the form of the following differential equation:

$\frac{\mathrm{d}}{\mathrm{d} t} p(t)=p(t) R$

where $R$ is a square matrix of the transfer rates.

- It can be shown that for such a Markov process, the time spent in state $\mathrm{i}$ before making a transition to state $j$ is negative exponentially distributed with mean $1 / \lambda_{i j}$.

- It may be noted that negative exponential distribution has the same forgetfulness as the Markovian assumption.

- A system with $S$ possible states will have $S^{2}$ differential equations which are too large a number to solve easily. One therefore works with state probabilities.

- A state probability $P_{j}(t)$ is defined as the probability that the system is in state $j$ at time $t$ (no matter in what state it was $\Delta t$ time back). Thus it is the sum of the probabilities of transition from state $i$ to $j$ over all states $i j$.

$P_{j}(t)=\sum_{i=1}^{S} p_{i j}(t)$ 
The instantaneous rate of change of state probabilities can be derived as,

$\frac{\mathrm{d}}{\mathrm{d} t} P_{j}(t)=\sum_{i=1}^{S} P_{i}(t) \lambda_{i j}$

Defining $P(t)$ as the row vector of state probabilities, these state equations can be obtained in the following vector matrix form:

$\frac{\mathrm{d}}{\mathrm{d} t} P(t)=P(t) \times R$

Taking transpose of both sides of Eq. (8) and defining $\underline{Z}(t)=P^{T}(t)$,

and $R=R^{T}$, Eq. (8) becomes,

$\underline{Z}=R \underline{Z}(t)$

This is the familiar vector matrix state differential equation of an autonomous linear system. Thus it can be concluded that stationary, continuous-time Markov processes are representatives of autonomous linear systems.

Development of equivalent system dynamics model

In this section, a system dynamics model has been developed which is equivalent to continuous-time Markov process as described below.

Equation (7) shows that the instantaneous rate of change of $j$ th state probability,

$\frac{\mathrm{d}}{\mathrm{d} t} P_{J}(t)=\sum_{i=1}^{S} P_{i}(t) \lambda_{i j}$

This can be written as

$\frac{\mathrm{d}}{\mathrm{d} t} P_{j}(t)=P_{j}(t) \lambda_{j j}+\sum_{i=1, \mathrm{i} \neq \mathrm{j}}^{S} P_{i}(t) \lambda_{i j}$

Making use of Eq. (4), the above equation can be written as follows:

$\frac{\mathrm{d}}{\mathrm{d} t} P_{j}(t)=P_{j}(t)\left\{-\sum_{k=1, \mathrm{k} \neq \mathrm{j}}^{S} \lambda_{j k}\right\}+\sum_{i=1, \mathrm{i} \neq \mathrm{j}}^{S} P_{i}(t) \lambda_{i j}$

$\frac{\mathrm{d}}{\mathrm{d} t} P_{J}(t)=\sum_{i=1, \mathrm{i} \neq \mathrm{j}}^{S} P_{i}(t) \lambda_{i j}-\sum_{k=1, \mathrm{k} \neq \mathrm{j}}^{S} P_{j}(t) \lambda_{j k}$

Immediately the above Eq. (13) can be recognized as a level equation with $P_{j}$ as a level variable, and $\sum_{i=1, \mathrm{i} \neq \mathrm{j}}^{S} P_{i}(t) \lambda_{i j}$ as the total inflow into the level during the period $t$ and $t+\Delta t$.

During the same period, $\sum_{k=1, \mathrm{k} \neq \mathrm{j}}^{S} P_{j}(t) \lambda_{j k}$ is the total flow out of the level.
The inflow increases the probability $P_{j}(t)$ due to transitions to state $j$, while the outflow reduces $P_{j}(t)$ due to transitions out of the state $j$.

The transfer rates $\lambda_{i j}$ and $\lambda_{j k}$ are the constants associated with the input and output rates.

It can be observed that rates are linearly dependent on level variables from which they emerge. Thus stationary, continuous-time Markov models are algebraically equivalent to linear system dynamics models.

\section{A MSD approach to system reliability/availability assessment}

The procedure that we propose in this paper [Srinivasa Rao and Naikan (2008a, b, c, 2009a, b, c, 2010, 2011)] to develop the availability/reliability study using continuoustime system dynamics simulation is described in Table 1, where we distinguished a total of five steps.

Step 1: (System's states description). The first step of the study is the identification of the states of the system that means the selection/determination of the system's or components within the system functional or up and down states blocks, and how they relate to each other. As a result of this step, we will obtain a state transition diagram of the system that contains the relations among its components' states and their reliability features.

Step 2: (Data collection). Before starting to build the simulation model in step 3 , we need to know the design, the complete taxonomy of components of the system, and we will try to find out full reliability and maintainability information of each item in terms of components' failure rate and repair date data information. There are several sources to find this information such as public data books, databanks, performance data from the actual plant, expert judgments and laboratory testing.

Step 3: (Building the system dynamics simulation model). In this step, the state transition diagram of the system will be converted into equivalent MSD model. The resulting model is called as system dynamics model.

Step 4: (MSD simulation): In simulating the MSD model, an algorithm written in $\mathrm{C}$ was developed and it is used on a digital computer for developing various scenarios and to obtain the required simulation replications and the results.

Step 5: (Results and analysis). This step will include the presentation of result for the availability and reliability parameters corresponding to the functions of our interest in the different configurations. This step implies explaining the results obtained with the simulation, and the factors that may lead to those results, and also providing possible 
Table 1 Steps in the availability/reliability assessment

\begin{tabular}{|c|c|c|}
\hline Step name & Description & Result \\
\hline System's states description & $\begin{array}{l}\text { Determination of the basic functional states for the } \\
\text { system configuration and for every function to } \\
\text { analyze }\end{array}$ & $\begin{array}{l}\text { List of all functional states. State transition diagram of the } \\
\text { system that contains the relations among states and their } \\
\text { reliability features }\end{array}$ \\
\hline Data collection & $\begin{array}{l}\text { Compilation of the necessary reliability and } \\
\text { maintenance data (and information) for each one } \\
\text { of the considered states }\end{array}$ & $\begin{array}{l}\text { Reliability and maintenance data from one state to another: } \\
\text { failure rate, repair rate, MTTR, MTBF, MTTM, preventive } \\
\text { schedule, times, etc }\end{array}$ \\
\hline Model building & $\begin{array}{l}\text { Continuous-time system dynamics simulation } \\
\text { model building }\end{array}$ & Markov system dynamics model \\
\hline $\begin{array}{l}\text { System dynamics } \\
\text { simulation }\end{array}$ & Simulation scenarios and experiments design & $\begin{array}{l}\text { Scenario listings, required simulation replications and } \\
\text { the results, etc. }\end{array}$ \\
\hline Results and analysis & $\begin{array}{l}\text { Simulation results calculation } \\
\text { Simulation results discussion }\end{array}$ & $\begin{array}{l}\text { Result of the parameters of availability and reliability of the } \\
\text { functions of our interest in the different configurations } \\
\text { Interpretation of results and their discussions }\end{array}$ \\
\hline
\end{tabular}

actions to improve system's availability or reliability to meet system's functional requirements.

\section{Reliability assessment of a standby system with repair}

The approach of system dynamics was created and developed in the late 1950s by a group of researchers led by Forrester at the Massachusetts Institute of Technology (MIT), Cambridge, MA [Forrester (1961)]. It is a methodology for modeling and redesigning manufacturing, business, and similar systems that are part man, part machine [Richardson (1999)]. It builds on information feedback theory, which provides symbols for mapping systems in terms of diagrams and equations, and a programming language for conducting computer simulations. Another advantage of system dynamics modeling is that it is easy to experiment with alternative values of parameters. Finally, the steady state solutions for these problems can be obtained easily by the inspection of the flow diagrams. The present work proposes MSD approach for reliability modeling of a standby system with repair as described in the following sections:

\section{Step 1: System's states description and assumptions}

In this system, the following assumptions are used to analyze the system through Markov analysis.

- The system consists of two components such that one is in online and another is in standby mode.

- The standby unit will have a reduced failure rate while in its standby mode.

- Once active, the standby (backup) unit may experience the same failure rate as the online (primary) system (if they are identical units) or may have a different failure rate. The dependency arises because the failure rate of the standby unit depends on the state of the primary unit.

- Repair of the primary unit is feasible when it is in a failed state, and the system will continue to operate as long as the backup unit has not failed.

- If the primary unit is restored before the backup has failed, then the system's perspective no failure has occurred and the system returns to its initial state.

- The primary unit has a failure rate $\lambda_{1}$ and constant repair rate ' $\mu$ ' whereas the standby unit is having a failure rate $\lambda_{1}$ when it is in online and a failure rate $\lambda_{2}^{-}$ when it is in standby mode.

- The failure of the secondary unit while in standby remains undetected and therefore no repair is possible.

The rate diagram for Markov analysis is shown in Fig. 1. In this diagram, state 1 indicates that the primary unit is in operating mode and the second (backup) unit is in standby mode, state 2 indicates that the backup unit is in operating mode and the primary unit under repair, and state 4 indicates that backup unit is in the failed state. And the state 3 represents a failure (perhaps undetected) of the standby unit while in standby with $\lambda_{2}^{-}$being the corresponding failure rate.

In the Markov analysis, the system reliability is established from system state probabilities which are evaluated using a rigorous mathematical treatment as discussed by several authors. However, in the proposed model, the system state probabilities are established by observing the dynamic behavior of the system over its entire simulated mission period using the system dynamics approach. This approach is as discussed in the following section. 


\section{Step 2: Data collection}

The proposed MSD methodology starts after identification of the system states as mentioned in the previous section. To illustrate the above system, let us consider the following example which consists of the required data for further analysis.

Let us take an active generator which has a failure rate (failures per day) of 0.01 and a repair rate of 0.10 . An older standby generator has a failure rate of 0.001 while in standby and a failure rate of 0.10 when online. Now it is required to solve for the system reliability for a planned 30-day use. This solution takes place as follows. This illustrative example is as similar to the system under study, i.e., a standby system with repair. The data regarding the constant failure and repair rates of this illustrative example have been used in the proposed MSD simulation model.

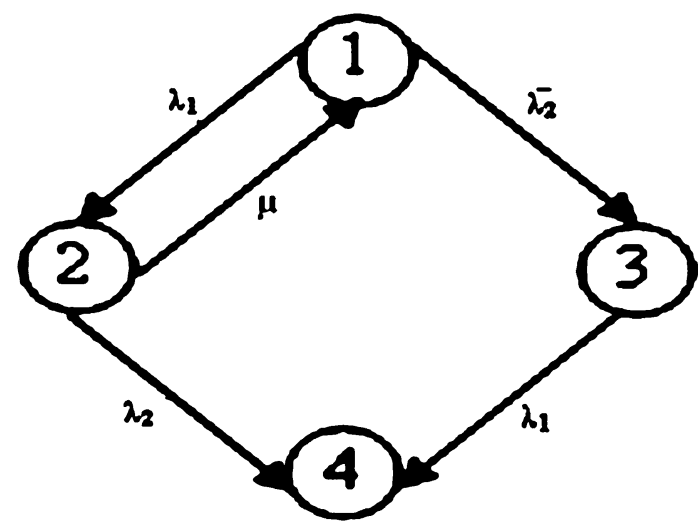

Fig. 1 Rate diagram for a standby system with repair
Step 3: Building the system dynamics simulation model

The next step in the modeling process is to convert the rate diagram of system into the rate and level diagrams. The rate diagram (Fig. 1) of the standby system with repair is now converted into a comprehensive system dynamic model. This is presented in Fig. 2.

In the model depicted in Fig. 2, the four states of the standby system with repair are indicated with level variables S1, S2, S3, S4 and the state transitions are indicated with rate variables (R12, R21, R13, R34, R24) with the corresponding transition rates $\left(\lambda_{1}, \lambda_{2}, \mu, \lambda_{2}^{-}\right)\left(\lambda_{1}\right.$ indicates failure rate of primary unit and $\mu$ indicates its repair rate, $\lambda_{2}$ indicates failure rate of the standby unit when it is in online and $\lambda_{2}^{-}$indicates failure rate of the standby unit when it is in standby mode). The initial value of system reliability (as indicated at the level variable S1) is assumed as unity. The level of system reliability will be decreased by the rate of failures of either the primary unit or standby unit and it will be recovered with the repair of the primary unit before the standby unit has failed. These are measured as probability density functions (pdfs) of the system. The rate variables are influenced by the respective auxiliary variables, i.e., failure rate and repair rates of the primary unit and the failure rate of the standby unit along the entire mission or operating time. In addition, the level of failure state of the system (i.e., level variable S4) is increased by these rate variables of the primary and standby units, leading to the declining reliability of system. Figure 2 depicts the basic structure of the standby system with repair reliability model.
Fig. 2 A comprehensive system dynamics model

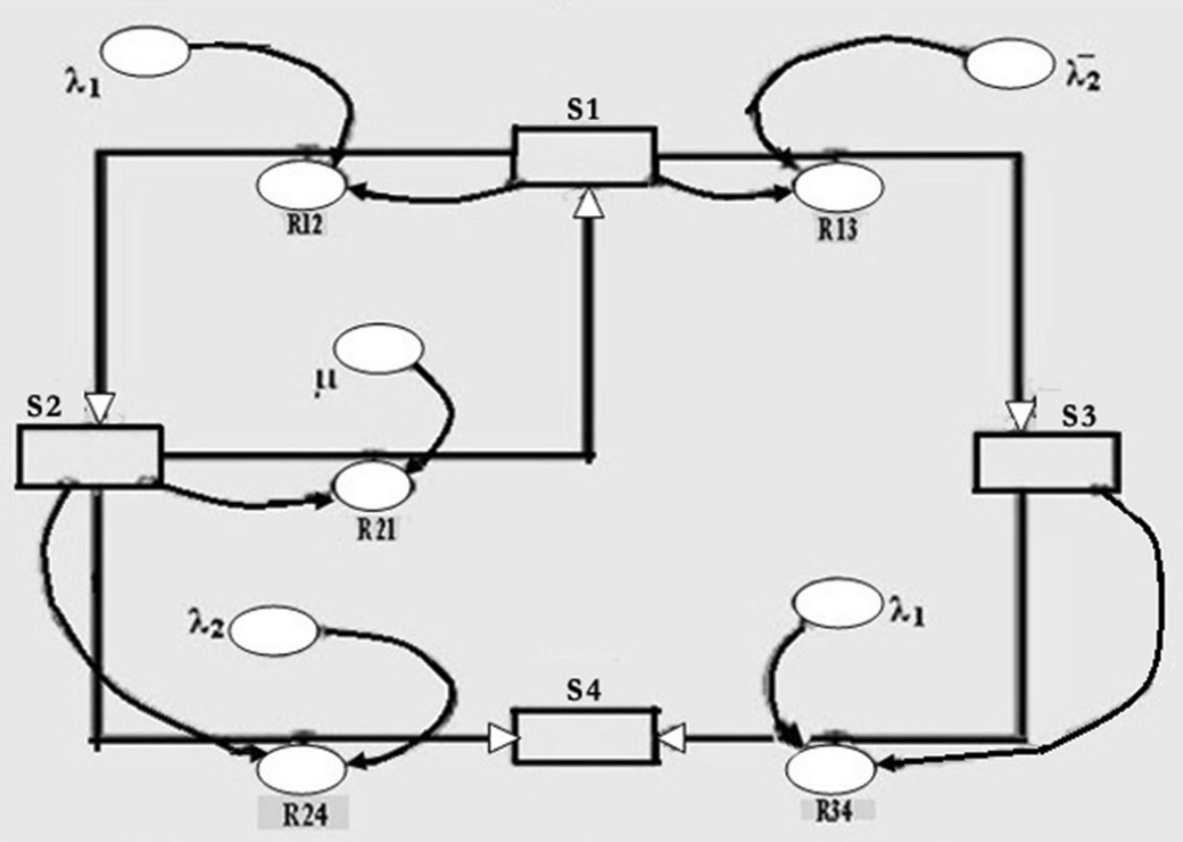


Fig. 3 (State S1 vs. time) performance graph

Fig. 4 (State S1, S2, S3 vs. time) performance graph

Fig. 5 (States S1, S2. S3, S4 vs. time) performance graph
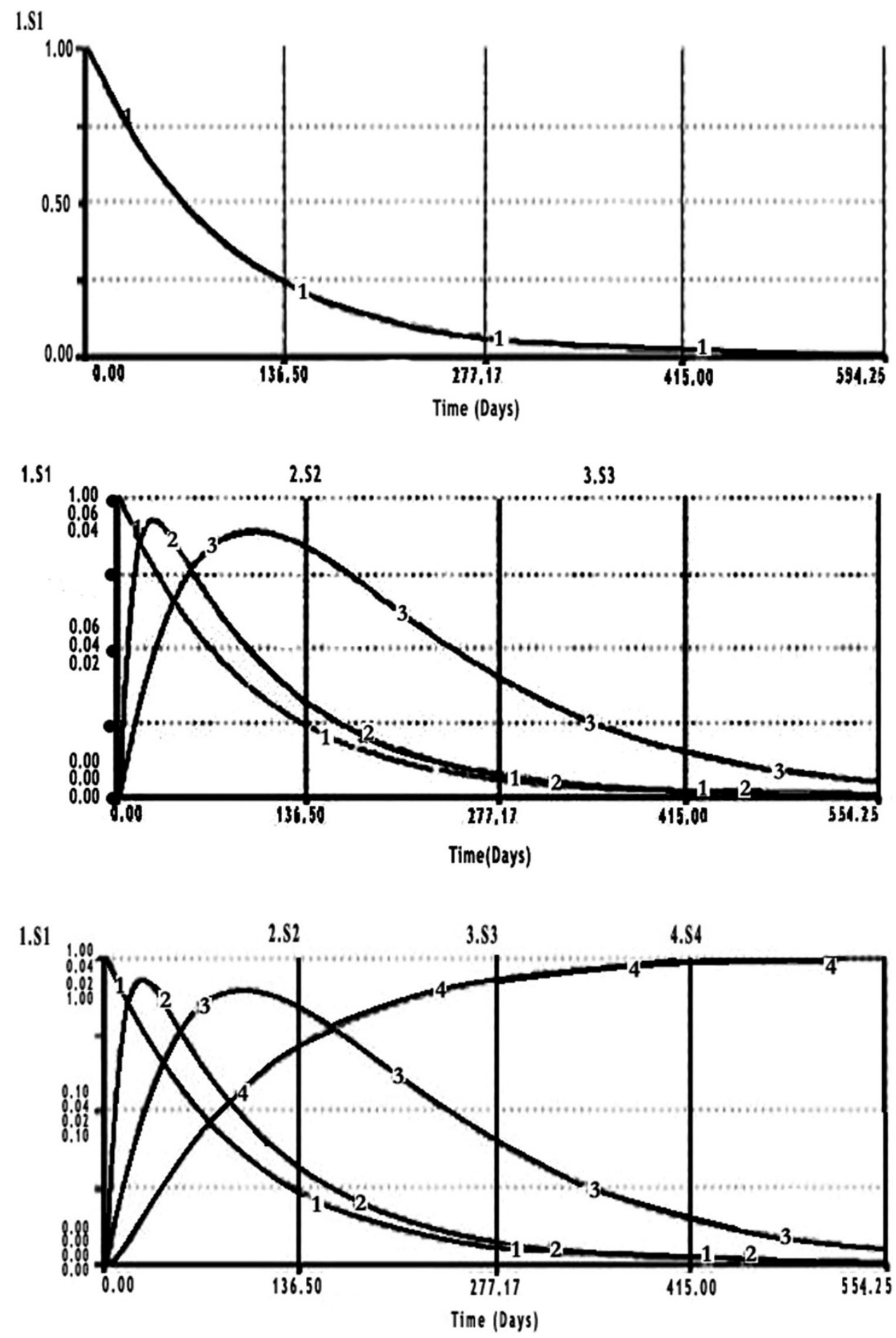

Step 4: System dynamics simulation

The next stage of MSD approach is to simulate the comprehensive MSD model of the system using the proposed algorithm. Using this, the state probabilities of the system have been calculated for reliability analysis and also the required simulation can be performed to study the dynamic behavior of the system as follows.
Proposed algorithm

Step 1 The values of transition rates $\left(\lambda_{1}, \lambda_{2}, \mu, \lambda_{2}^{-}\right)$and the time interval $d t$ are taken as inputs. Also the total time $T$ is taken as input, i.e., the time for which the system has to be simulated.

Step 2 Initially set $\mathrm{S} 1$ equal to one.

Step $3 \quad$ Set $\mathrm{S}_{i}$ equal to zero for $i=2,3,4$. 
Step 4 A conditional loop is formed with the condition, $t<T$.

Step 5 In each execution of the loop, the time is increased by $\mathrm{d} t$, i.e., $t=t+\mathrm{d} t$.

So, the loop will continue till time $T$ with each step taken at time difference $\mathrm{d} t$.

Step 6 As assumed S1 initially has probability unity, and the system fails when it becomes zero. Run a conditional loop as long as the condition, i.e., the probability of $\mathrm{S} 1>0$ is satisfied.

Step 7 Within the loop all the rate variables are calculated. The probabilities of the states are calculated first and the outflow rates are calculated according to the logic as follows:

$$
\begin{aligned}
& S 1(t)=S 1(t-\mathrm{d} t)+(R 21-R 12-R 13) * \mathrm{~d} t \\
& S 2(t)=S 2(t-\mathrm{d} t)+(R 12-R 21-R 24) * \mathrm{~d} t \\
& S 3(t)=S 3(t-\mathrm{d} t)+(R 13-R 34) * \mathrm{~d} t \\
& S 4(t)=S 4(t-\mathrm{d} t)+(R 34+R 24) * \mathrm{~d} t
\end{aligned}
$$

Step 8 Then all the required values are displayed and the required graphs can be drawn using these values to study the dynamic behavior.

Table 2 System reliability values

\begin{tabular}{lll}
\hline Time (days) & $\begin{array}{l}\text { Reliability by } \\
\text { proposed model (Rs) }\end{array}$ & $\begin{array}{l}\text { Reliability by } \\
\text { conventional method (Rc) }\end{array}$ \\
\hline 0 & 1.000 & 1.000 \\
5 & 0.9900 & 0.9896 \\
10 & 0.9701 & 0.9718 \\
15 & 0.9308 & 0.9309 \\
20 & 0.9003 & 0.9036 \\
25 & 0.8509 & 0.8512 \\
30 & 0.8218 & 0.8226 \\
\hline
\end{tabular}

\section{The model experimentation}

By implementing this algorithm, the model experimentation can be performed as follows.

The simulation of the proposed model confirms that the operating state (reliability) of the standby system with repair decreases with increase in hazard rates as shown in Fig. 3. The following experiments have been performed in this study:

Experiment 1: Test simulation run The system was first simulated with a test simulation run with an assumption that initially it is having maximum reliability, i.e., unity and the primary and standby units of the system are in operating state (i.e., at level variable S1). And the operating state of the system reaches its minimum level with increase in time.

Experiment 2: Second simulation run In a second simulation run, the system has been simulated (for 554.25 days) with the given values of failure rate and repair rates of the primary unit and the failure rates of the standby unit when it is in online as well as in standby mode. In this, the operating state (i.e., reliability as indicated with level variable S1) of the system reaches its minimum level with increase in time as shown in Fig. 3.

Experiment 3: Third simulation run In a third run, the proposed model for the standby system with repair has been simulated (for 554.25 days) by considering the state 2 (i.e., S2) which indicates that the backup unit is in operating mode and the primary unit under repair and also the state 3 (i.e., S3) which represents a failure (perhaps undetected) of the standby unit while in standby with $\lambda_{2}^{-}$being the corresponding failure rate as shown in Fig. 4.

Finally, the system has been simulated simultaneously by considering all of its states to analyze its reliability. Figure 5 depicts the dynamic behavior of the standby system with repair in its all states. The simulation results in

Fig. 6 Performance graph

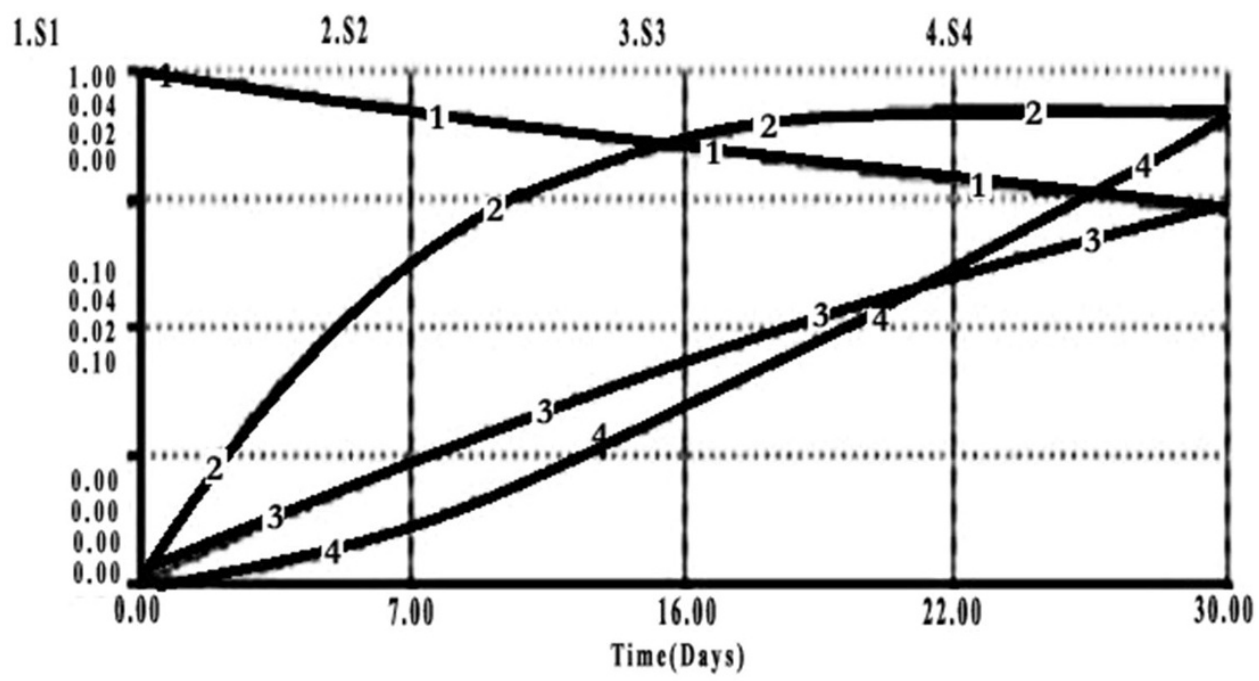




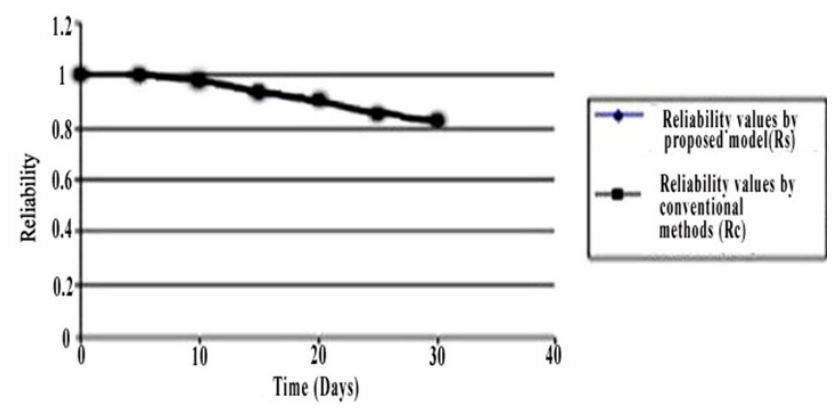

Fig. 7 Comparison of results

Fig. 5 clearly indicate that the operating state of the system decreases with increase in time due to primary and standby unit's failures. And also the system will be in operating state if one of its units is in operating state (S2, S3 curves indicate this in Fig. 5). The system reaches its failed state when both of its units fail. And the sum of S1, S2, and S3 state probabilities will give the system reliability.

\section{Results and discussion}

The system considered in this work can be solved using the conventional Markov analysis. But it will consist of so many Kolmogorov system of differential equations to calculate the reliability. There is much difficulty in solving these equations. And also, the system reliability is established from system state probabilities which are evaluated using a rigorous mathematical treatment. So, to simplify this analysis, the same system has been considered in this paper and solved using the proposed model as described earlier to calculate the reliability and also the dynamic behavior of the system. The obtained results are presented in Table 2; Figs. 6 and 7 which clearly indicate that the simulated results very closely match with computed values. The authors have carried out similar studies for this proposed model and simulated the model for a longer period (554.25 days) to study the dynamic behavior of the system till its reliability approaches zero. In this way, the proposed model approach can be used for analyzing more than one standby or for analyzing more number of states of standby systems.

It is clear from the results, the proposed system dynamics modeling frame work can be used as an alternative approach to analyze the reliability of systems instead of using the Markov methodology alone which requires too many mathematical equations and calculations with unrealistic assumptions. It is worth mentioning here that the MSD modeling is much easier compared to the traditional approaches to study the reliability of complex systems. Conclusively, for analyzing the dynamic behavior of systems, MSD simulation seems to be useful and also to understand the functioning and interaction of the different facets of reliability of systems. It can be stated that a switch from conventional methods to simulation seems to be the most promising reliability modeling and analysis strategy for systems.

\section{Conclusions}

In this paper, a hybrid approach called as MSD approach has been proposed for reliability analysis and to study the dynamic behavior of systems. The proposed framework is illustrated for a standby system with repair. The results of the simulation when compared with those obtained by traditional Markov analysis clearly validate the proposed approach as an alternative approach for reliability analysis. The procedure for the development of the MSD approach for this system is explained and the model is run to observe all of its states. The proposed methodology is applicable for all types of failure rates and repair rates and it is much simpler compared to traditional approaches. Further, this methodology can be used for studying various scenarios having managerial implications of system reliability. It is important to note that reliability declining of component or system has to be observed carefully to achieve the desired results. Managers must beware of the existing interdependencies within the component or system. Accordingly, the model can be used as a simulation tool. Based on simulation analyses, managers can learn how to deal with such a comprehensive approach like the one investigated in this paper. And also, the different parties, i.e., engineers and machine operators, can jointly work with the model to understand the dynamic behavior of systems.

Open Access This article is distributed under the terms of the Creative Commons Attribution License which permits any use, distribution, and reproduction in any medium, provided the original author(s) and the source are credited.

\section{References}

Islam A, Tedford D (2012) Risk determinants of small and medium sized manufacturing enterprises (SMEs): an exploratory study in New Zealand. J Ind Eng Int 8:12. doi:10.1186/2251-712X-8-12 Forrester JW (1961) Industrial dynamics. MIT Press, Cambridge

Goel LR, Gupta R, Shrivastava P (1990) Profit analysis of a two unit cold standby system with imperfect switch and varying physical condition of the repairman. Microelectr Reliab 30(4):655-660

Gupta R, Goel LR (1991) Profit analysis of a two multicomponent unit standby system with MRT. Microelectr Reliab 31(1):7-10

Gupta R, Mumtaz SZ (1996) Stochastic analysis of a two unit cold standby system with maximum repair time and correlated failures and repairs. J Qual Maint Eng 2(3):66-76

Khalaj M, Khalaj F, Khalaj A (2013) A novel risk based analysis for the production system under epistemic uncertainty. J Ind Eng Int 9:35. doi:10.1186/2251-712X-9-35 
Lal AK, Gupta P, Sharma RK, Singh J (2005) Numerical analysis of reliability and availability of the serial processes in butter oil processing plant. Int J Qual Reliab Manag 22(3):303-316

Moafib SE, Goel LR, Gupta R (1991) Comparison of two stochastic models for two unit series system with cold standbys. Microelectr Reliab 31(6):1105-1111

Mokaddis GS, Tawfek ML, Elhssia SAM (1997) Cost analysis of a two dissimilar unit cold standby redundant system subject to inspection and two types of repair. Microelectr Reliab 37(2):335-340

Mousavi SM, Makui A, Raissi S, Mojtahedi SMH (2012) A multicriteria decision making approach with interval numbers for evaluating project risk responses. Int J Eng Trans B 25(2):121-129

Raissi S, Gatmiry Z (2012) A six sigma approach to boost up time domain reliability in multi stage services. Afr J Bus Manag 6(4):1367-1374

Richardson GP (1999) Reflections for the future of system dynamics. J Oper Res Soc 50(4):440-449

Song SB, Deng YL (1993) Reliability analysis of a three unit system in a changing environment. Microelectr Reliab 33(5):637-640

Srinivasa Rao M, Naikan VNA (2008a) A Markov system dynamics (MSD) approach for reliability analysis of systems. Int $\mathrm{J}$ Commun Dependab Qual Manag 11(3):17-30

Srinivasa Rao M, Naikan VNA (2008b) A system dynamics based soft computing approach for reliability analysis of systems. In: Proceedings of the International conference ORSI, S.V. University, Tirupati, India, 62, RLT03

Srinivasa Rao M, Naikan VNA (2008c) A soft computing approach for reliability analysis of repairable systems. In: Proceedings of the international conference ICAME, S. V. National Institute of Technology, Surat, India, pp 18-36

Srinivasa Rao M, Naikan VNA (2009a) A managerial tool for reliability analysis using a novel markov system dynamics (MSD) approach. Int J Manag Sci Eng Manag 4(3):230-240

Srinivasa Rao M, Naikan VNA (2009b) A novel Markov system dynamics framework for reliability analysis of systems. Int $\mathbf{J}$ Qual Reliab 24(1):101-116

Srinivasa Rao M, Naikan VNA (2009c) A Markov system dynamics (MSD) based availability and reliability analysis of a process industry. Int J Commun Dependab Qual Manag 12(3):26-49
Srinivasa Rao M, Naikan VNA (2010) A system thinking approach for time dependent availability analysis of multi component systems. In: Proceedings of the international conference on reliability, safety and hazard. Navi Mumbai, India, December $14-16,2010$

Srinivasa Rao M, Naikan VNA (2011) A hybrid Markov system dynamics approach for availability analysis of degraded systems. In: Proceedings of the 2011 International conference on industrial engineering and operations management, Kuala Lumpur, January 22-24, 2011

Zhang YL, Lam Y (1996) Analysis of a two component series system with a geometric process model. Nav Res Logist 43:491-502

M. Srinivasa Rao is a Professor in GMR Institute of Technology, Rajam, India. He took his Doctorate degree in Reliability engineering from Reliability engineering centre at Indian Institute of Technology, Kharagpur, India. He received his B.E in Mechanical Engineering and M.E in Industrial Engineering from Andhra University, Visakhapatnam, India. He has published his research works in many international journals and presented papers in conferences. His current research interests include condition monitoring, mechanical system reliability, probabilistic risk and system assessment, quality planning and management, reliability and availability analysis and modeling of systems using simulation methodologies. Email: srinivas.m@gmrit.org.

V. N. A. Naikan is a Professor in Reliability engineering centre at Indian Institute of Technology, Kharagpur India. He took his Masters and Doctorate degree in industrial engineering and management with specialization in Quality and Reliability Engineering from Indian Institute of Technology, Kharagpur, India. He has worked with Indian Institute of Management, Ahmedabad, and Indian Space Research Organization, Ahmedabad, India. He has visited the Chinese University of Hong Kong for doing research. He has published his research works in many international journals, and presented papers in conferences. He is also actively involved in industrial consultancy and sponsored projects. His research interests include condition monitoring, mechanical system reliability, probabilistic risk and system assessment, quality planning and management, reliability of engineering systems. Email: naikan@hijli.iitkgp.ernet.in. 